\title{
Evolutionary Distance and Conserved Domain Analysis of Divergent Phylogenetic Lineages from Genus Naja
}

Sherkhane AS and Gomase VS*

The Global Open University, India

\begin{abstract}
Naja Naja is one of the poisonous snakes in the genus Naja of Elapids family; commonly called Indian cobras and are mostly found in Asia and Africa. They are highly venomous species having a cocktail of chemicals that have different effects may be speed the heart rate, blood pressure and interfere with the normal function of the nervous system and potently block $\alpha 7$ homo-oligomeric neuronal AChRs causing paralysis. The aim of the present study is to identify the origin of neurotoxin, prediction of structure and function of neurotoxin by multiple sequences analysis and observing the conserved pattern of amino acid residues and to construct the phylogenetic tree for organizing evolutionary history of $N$. Naja of genus Naja from Elapidae family.
\end{abstract}

Keywords: Neurotoxins; Naja genus; Multiple sequence alignment; Conserve domain; Phylogenetic analysis

\section{Introduction}

Naja Naja is one of the poisonous snakes in the genus Naja of Elapids family and commonly called Indian cobras and are mostly found in Asia and Africa [1]. Elapidae family approximately consists of 300 venomous snakes in 62 genera [2]. The genus Naja consists of currently 26 species of cobra of which 11 inhabit Asia and 15 occur in Africa [3,4]. Proteins from Naja Naja are potent postsynaptic neurotoxins [5]. Neurotoxins that acts by binding to the nicotinic acetylcholine receptors in the postsynaptic membrane of skeletal muscles [6] causing severe local pain, swelling immediately after bite; dark discoloration, necrosis, paralysis and even death [7-10]. In this research work, we study the origin and evolution of neurotoxin from $N$. Naja by multiple sequence alignments that provide the functional information of conserved sequence regions of neurotoxin from Naja Naja, phylogenetic analysis shows taxonomical classification, identifying and naming new members of protein families that derived from a common ancestor [11-15].

\section{Materials and Methods}

\section{Sources and sequence information of genus Naja neurotoxins}

Thirteen sepcies has taken from genus Naja of Elapidae family, in which targeted neurotoxins protein data were used to observe molecular resemble of related protein by phylogenic analysis (Table 1) $[16,17]$.

\begin{tabular}{|c|c|c|c|c|}
\hline Accession & Description & Identity $\%$ & E Value & Total Score \\
\hline P60814.1 & Naja naja & $100 \%$ & $5 \mathrm{e}-55$ & 176 \\
\hline Q9YGI4.1 & Naja atra & $98 \%$ & $1 \mathrm{e}-52$ & 170 \\
\hline O42255.1 & Naja sputatrix & $97 \%$ & $5 \mathrm{e}-52$ & 168 \\
\hline P82935.2 & Naja kaouthia & $88 \%$ & $2 \mathrm{e}-40$ & 139 \\
\hline Q9YGI2.1 & Naja naja & $72 \%$ & $4 \mathrm{e}-35$ & 125 \\
\hline P25679.2 & Naja kaouthia & $85 \%$ & $7 \mathrm{e}-31$ & 114 \\
\hline O93422.1 & Naja atra & $72 \%$ & $1 \mathrm{e}-27$ & 106 \\
\hline P01399.1 & Naja annulifera & $67 \%$ & $2 \mathrm{e}-24$ & 97.8 \\
\hline P85520.1 & Naja oxiana & $69 \%$ & $6 \mathrm{e}-24$ & 96.3 \\
\hline P25680.1 & Naja nivea & $68 \%$ & $5 \mathrm{e}-23$ & 94.0 \\
\hline P01401.1 & Naja haje haje & $62 \%$ & $3 \mathrm{e}-21$ & 89.4 \\
\hline P01400.1 & Naja melanoleuca & $62 \%$ & $2 \mathrm{e}-20$ & 87.0 \\
\hline Q9W717.1 & Naja naja & $54 \%$ & $8 \mathrm{e}-20$ & 86.3 \\
\hline
\end{tabular}

Table 1: Sequences producing significant alignments.

\section{Multiple sequence alignment of genus Naja neurotoxins}

Multiple sequence alignment [MSA] is conducted by COBALT, which aligns thirteen neurotoxin protein sequences of similar Naja genus using a combination of distance matrix and approximate parsimony methods. Numerical setting method is used to study the relative entropy threshold, in bits, that must be met for an alignment column to be displayed in red. A larger number indicates higher degree of conservation. The relative entropy is computed as: $\Sigma_{i} f_{i} \log 2\left(f_{i} / p_{i}\right)$, where $i$ is residue type, $f i$ is residue frequency observed in the multiple alignment column, and pi is the background residue frequency. Identity setting used for only columns with one residue type will be colored in red [18].

\section{Construction of a phylogenetic tree for neurotoxin from} genus Naja of Elapidae family

Phylogenetic analyses were performed by fast minimum evolution algorithm and Neighbor Joining algorithms to allow the reconstruction phylogenetic tree of the molecular evolutionary history of various aligned sequences that are useful to align highly evolved gene families clearing evolutionary relationships such as multiple actin proteins $[19,20]$. Trees were obtained by the methods fast minimum evolution algorithm and Neighbor Joining algorithms. Evolutionary distance is studied by Grishin (protein) model [21,22] and distance between two sequences modeled as expected fraction of amino acid substitutions per site given the fraction of mismatched amino acids in the aligned region and can be computed for fraction of mismatched amino acids larger than 0.75 [23-26].

\section{Results and Interpretation}

\section{Evolutionary distance}

This study, thirteen neurotoxin protein from genus Naja is

*Corresponding author: Gomase VS, The Global Open University, Nagaland India, Tel: 91-998-777-0696; E-mail: gomase.viren@gmail.com

Received May 21, 2014; Accepted August 20, 2014; Published August 25, 2014

Citation: Sherkhane AS, Gomase VS (2014) Evolutionary Distance and Conserved Domain Analysis of Divergent Phylogenetic Lineages from Genus Naja. J Data Mining Genomics Proteomics 5: 156. doi:10.4172/2153-0602.1000156

Copyright: (C) 2014 Sherkhane AS, et al. This is an open-access article distributed under the terms of the Creative Commons Attribution License, which permits unrestricted use, distribution, and reproduction in any medium, provided the original author and source are credited. 
Citation: Sherkhane AS, Gomase VS (2014) Evolutionary Distance and Conserved Domain Analysis of Divergent Phylogenetic Lineages from Genus Naja. J Data Mining Genomics Proteomics 5: 156. doi:10.4172/2153-0602.1000156

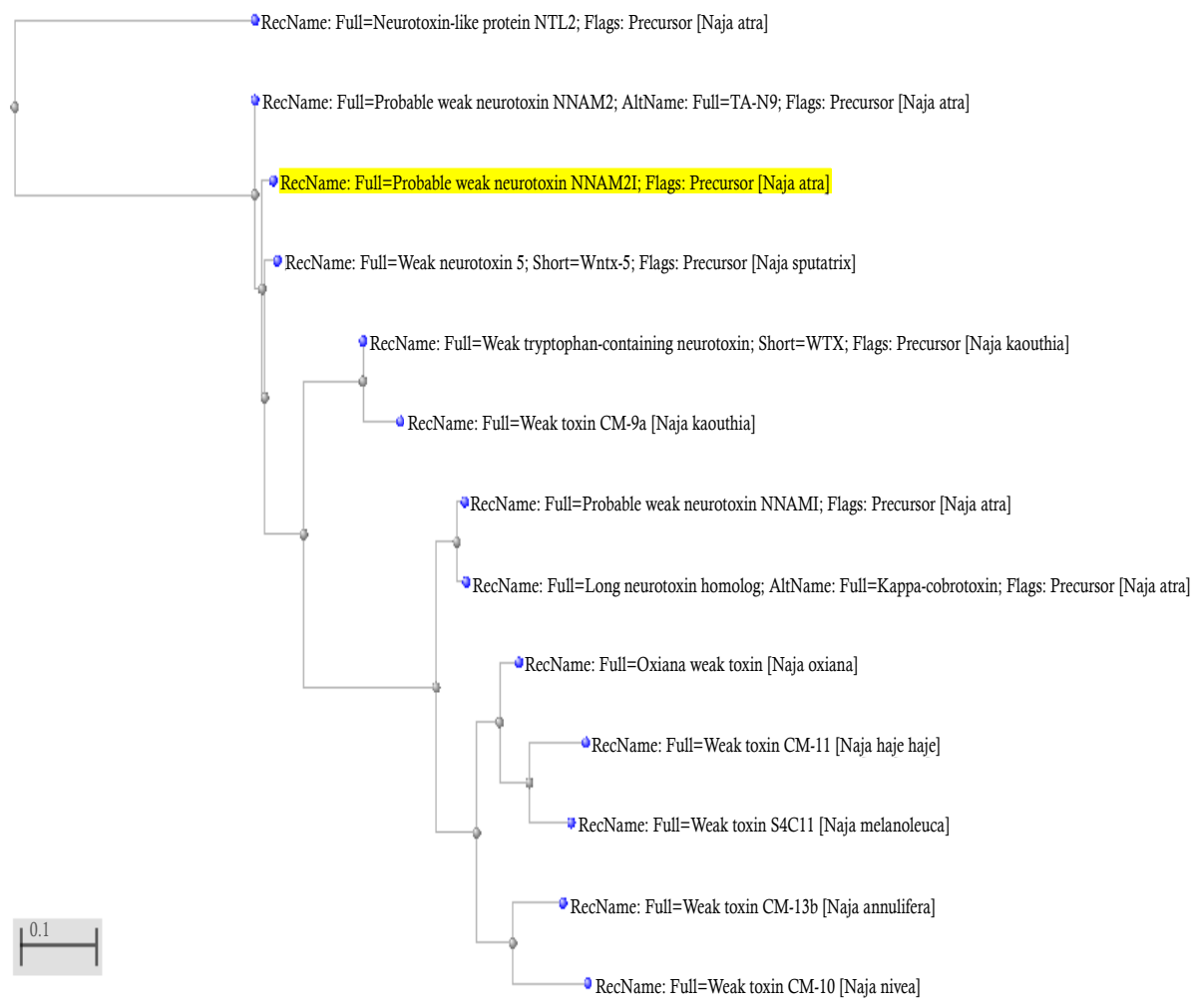

Figure 1: Rectangle tree - Fast minimum evolution algorithm - Phylogenetic study of genus Naja with the help of rendering tree showing the evolutionary difference with $N$. Naja in the rectangular shaped rooted tree, root is places in the longest edge.

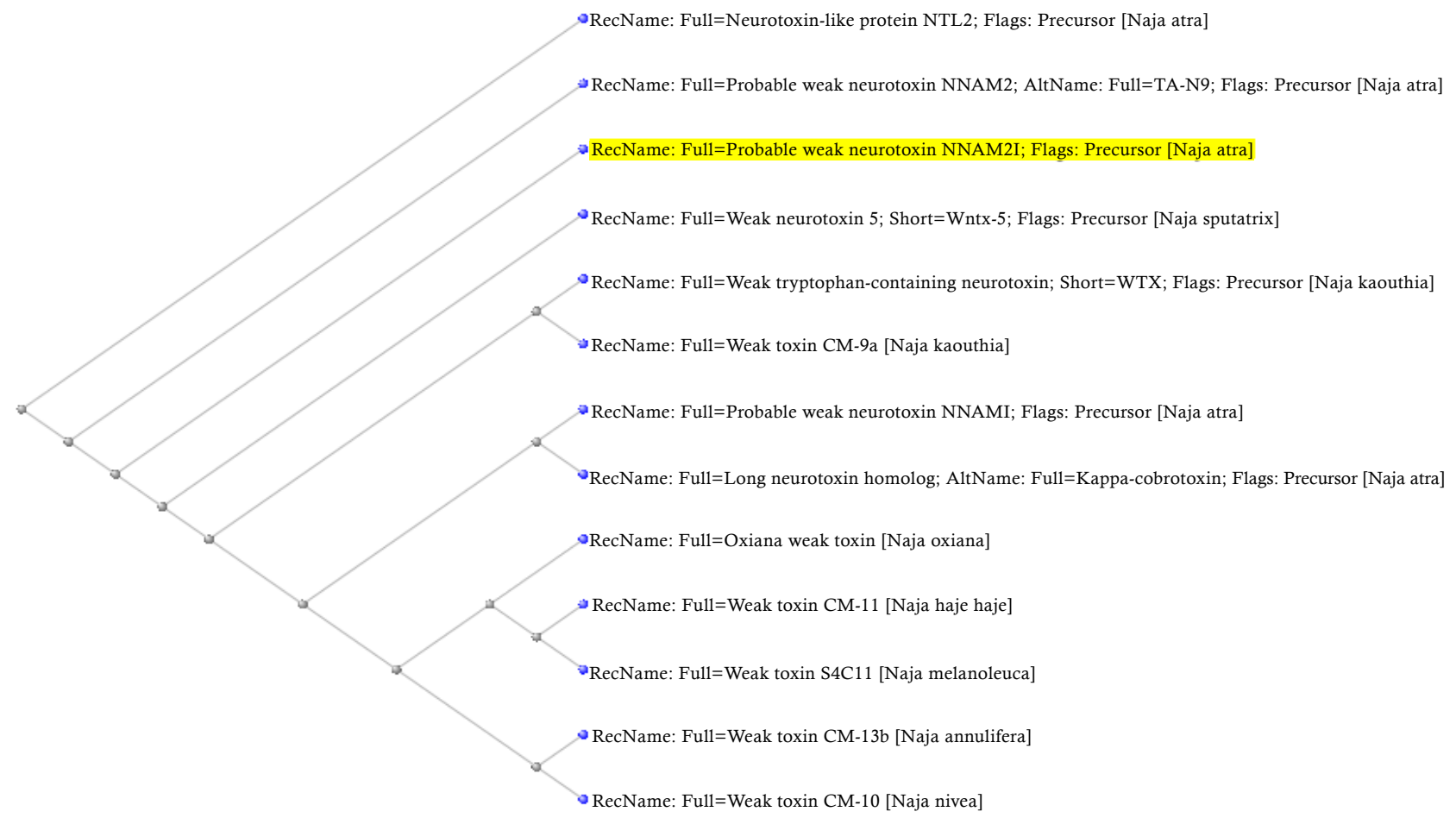

Figure 2: Slanted tree - Grishin (protein) model- Phylogenetic study of genus Naja with the help of rendering tree. Slanted tree is Similar to rectangle, but with triangular tree shape. 
summarized to study the evolutionary distance. The identification of the origin of neurotoxin protein from genus Naja, multiple sequences analysis, observing the conserved amino acid residues and reconstruct the phylogenetic tree specify the evolutionary history, relationship of N. Naja a with different species (Table 1). Rectangle tree shows rectangular shaped rooted tree, where root is places in the longest edge. Fast minimum evolution algorithm produce un-rooted tree such as ones shown as radial or force in the tabs below. The rooted trees are created by placing a root in the middle of the longest edge (Figures 1-4). Slanted tree shows similar to rectangle, but with triangular tree shape. Neighbour Joining algorithms produce un-rooted tree such as ones shown as radial or force in the tabs below. The rooted trees are created by placing a root in the middle of the longest edge.

\section{MSA}

Multiple sequence alignment analysis shows columns with no gaps are colored in blue or red. The red color indicates highly conserved regions and blue indicates less conserved ones. The Conservation analysis can be used to select a threshold for determining which columns are colored in red (Figure 5). Multiple sequence alignment identify conserved motifs and to predict functional role in the variable sites as well as conserved sites show the sequence divergence profile of these neurotoxin proteins, which demonstrate the sequence enrichment strategy of these sequences for adaptation to different physiological systems. Here we observed that from all sequences of neurotoxin proteins that Cys(c), Thr (T), Asn (N) (Hydrophilic amino acid) Phe(F), Gly(G), Ala(A), Pro(P) (hydrophobic amino acid), Lys $(\mathrm{K}), \operatorname{Arg}(\mathrm{R})$, Positive charged, $\operatorname{Asp}(\mathrm{D})$, Nagative charged which is conserved in all peptides having a common ancestor. That all of these peptides share eight highly conserved cysteines which were involved in the formation of $\beta$-strands are almost conserved. Cysteine (C) is conserved in all sequences at 8 sites. Multiple sequence alignment is carried out by COBALT of Naja genus.

\section{Conserved domain analysis}

Molecular study of N. Naja shows conserved domains and having one of snake toxin superfamily with user query added Superfamily (Figures 6 and 7). Snake toxin domains are present in short and long

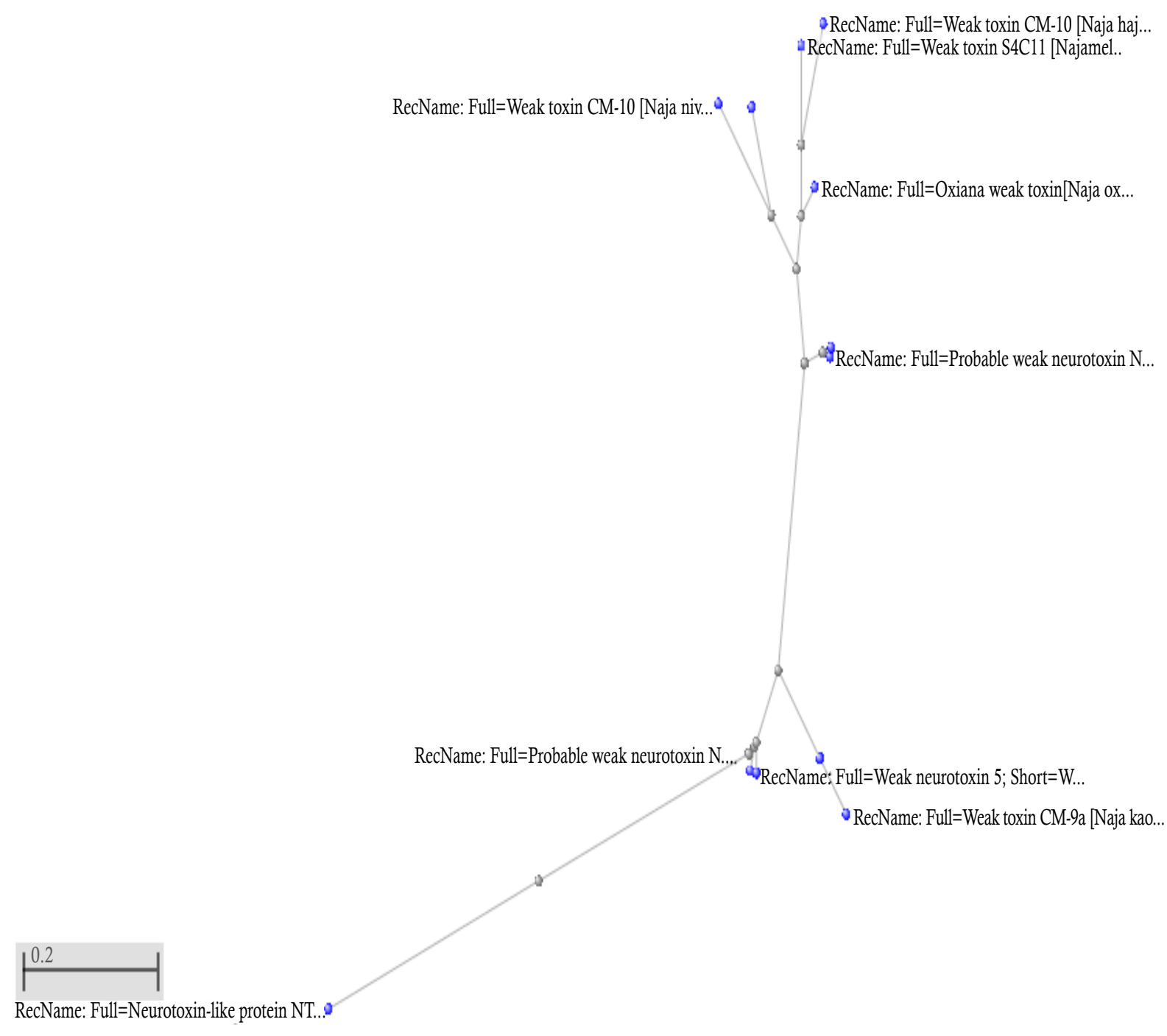

Figure 3: Radial tree - Grishin (protein) model- showing Phylogeny of genus Naja with the help of rendering tree. Radial tree is unrooted tree shape. 
Citation: Sherkhane AS, Gomase VS (2014) Evolutionary Distance and Conserved Domain Analysis of Divergent Phylogenetic Lineages from Genus Naja. J Data Mining Genomics Proteomics 5: 156. doi:10.4172/2153-0602.1000156

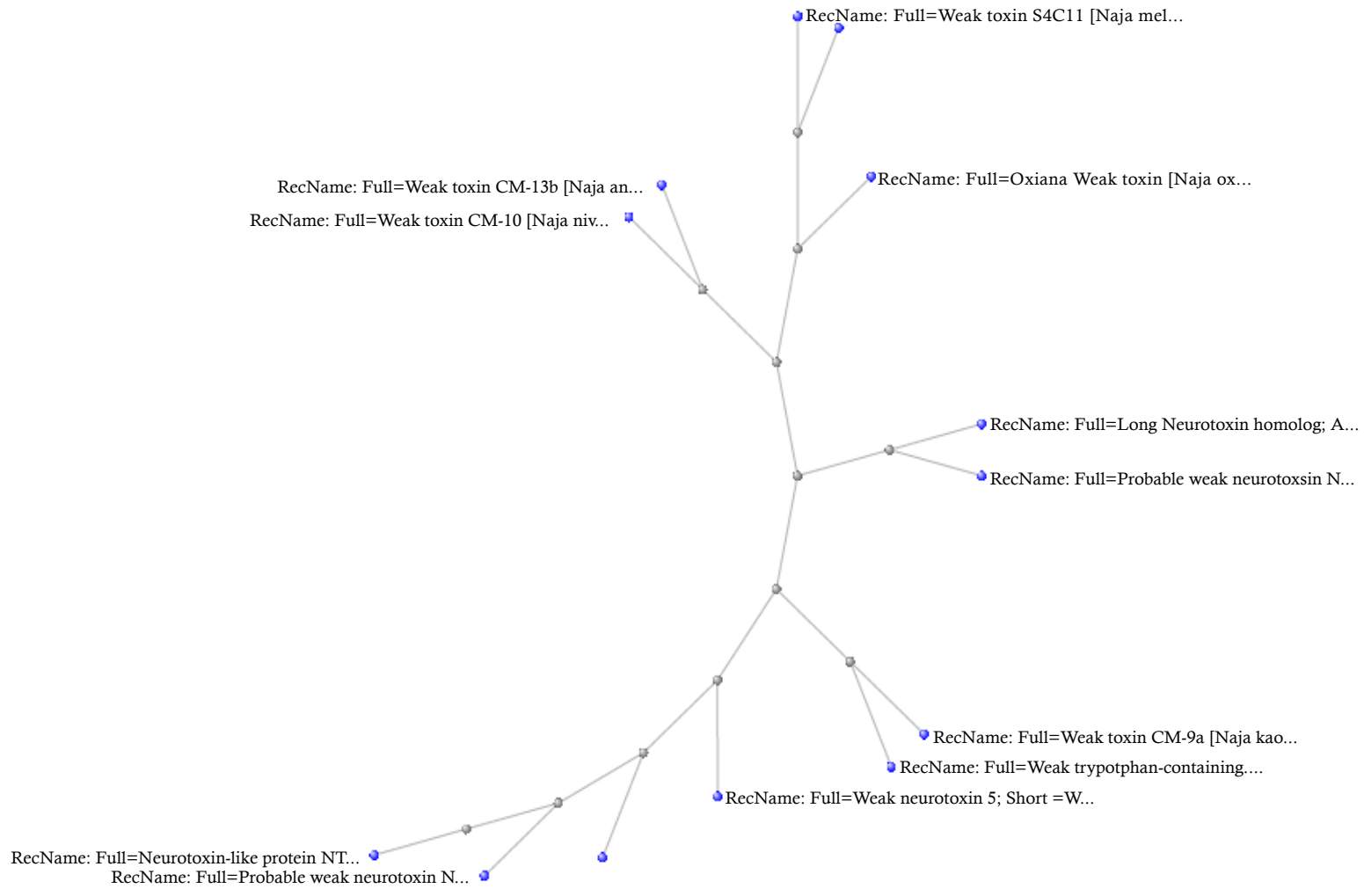

Figure 4: Force tree - Grishin (protein) model- showing Phylogeny of genus Naja with the help of rendering tree. Force tree is similar to radial, where nodes are pushed away from one another for better presentation.

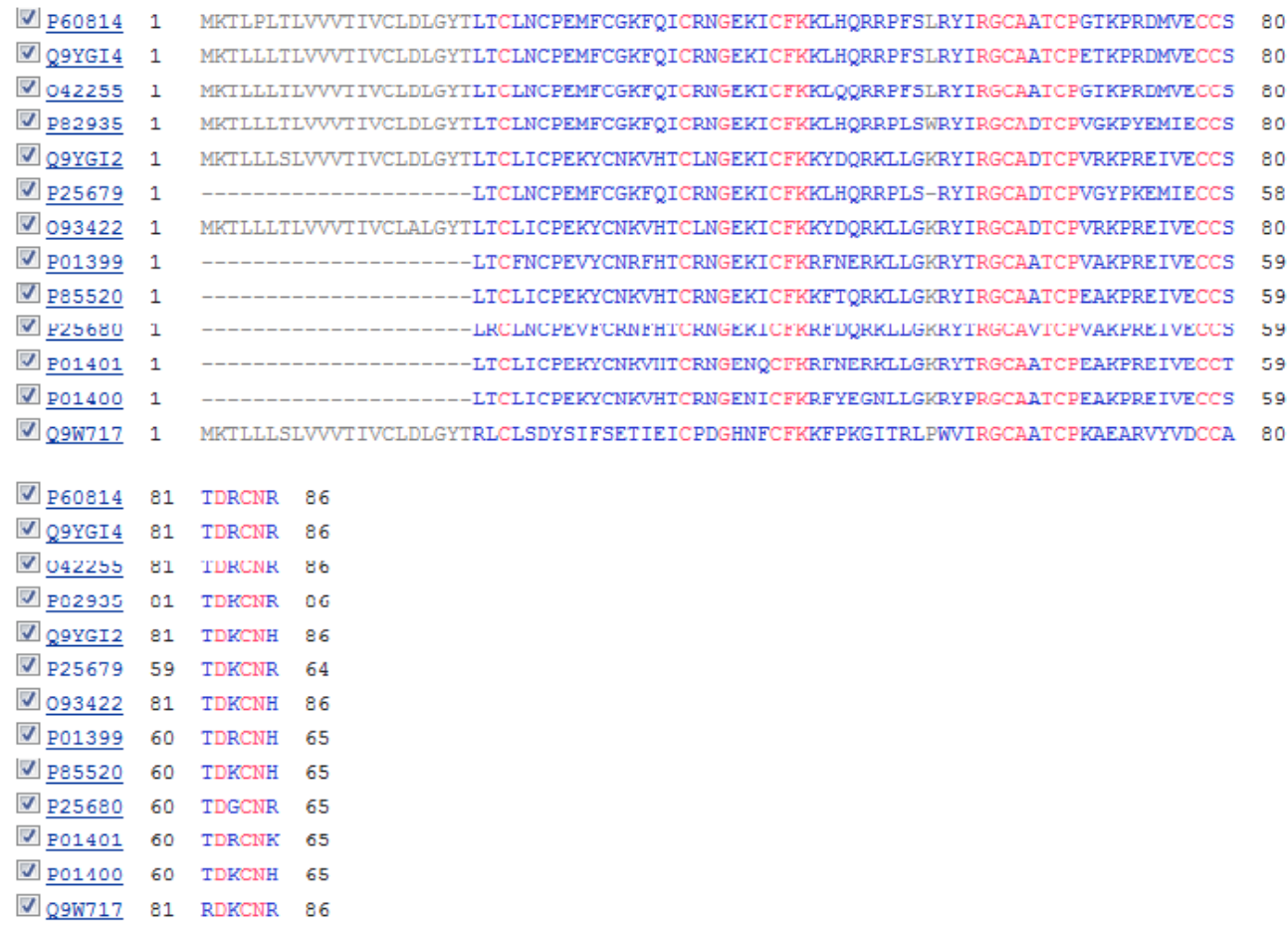

Figure 5: Multiple sequence alignment by COBALT of genus Naja. Here columns with no gaps are colored in blue or red. The red color Cys(c),Thr (T),Asn (N) hydrophilic polar, Phe(F),Gly(G),Ala(A),Pro(P) hydrophobic nonpolar,Lys (K),Arg(R), Positive charged, Asp(D),Nagative charge indicates highly conserved columns and blue indicates less conserved ones. The Conservation Setting can be used to select a threshold for determining, which columns are colored in red. 
Citation: Sherkhane AS, Gomase VS (2014) Evolutionary Distance and Conserved Domain Analysis of Divergent Phylogenetic Lineages from Genus Naja. J Data Mining Genomics Proteomics 5: 156. doi:10.4172/2153-0602.1000156

Page 5 of 6

\begin{tabular}{l|l} 
Query seq & \\
Specific hits & Toxin_l \\
Non-specific \\
hits \\
Superfanilies
\end{tabular}

Figure 6: Conserved domains on [gi|4165564|emb|CAA06888|] neurotoxin [N. Naja].

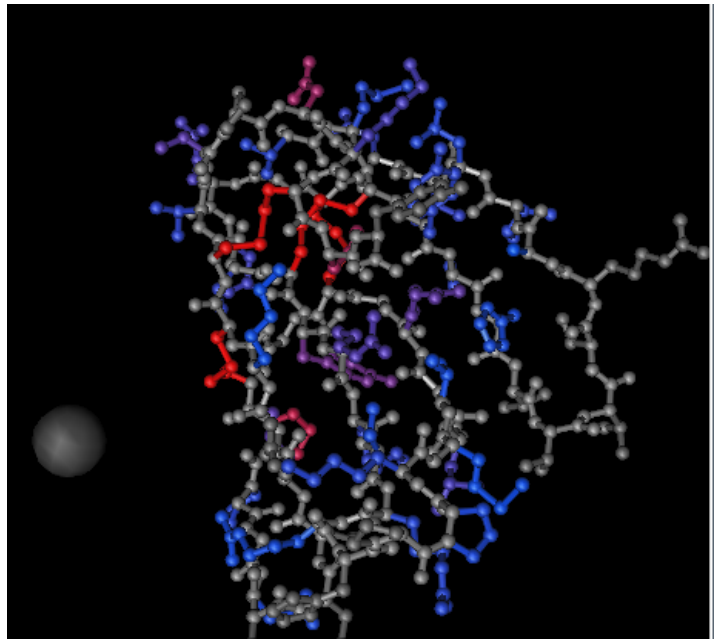

Figure 7: Sequence structure alignment shows similarity with structure of cl11586: snake_toxin Superfamily.

\begin{tabular}{|c|c|c|c|}
\hline Description & Pssmld & Multi-dom & E-value \\
\hline $\begin{array}{c}\text { snake_toxin[cd00206], Snake toxin domain, } \\
\text { present in short and long neurotoxins, cytotoxins } \\
\text { and short toxins, .. }\end{array}$ & 119411 & no & $8.44 \mathrm{e}-20$ \\
\hline $\begin{array}{c}\text { Toxin_1[pfam00087], Snake toxin; A family of } \\
\text { venomous neurotoxins and cytotoxins. Structure } \\
\text { is small, disulfide-rich, }\end{array}$ & 249576 & no & $5.93 \mathrm{e}-17$ \\
\hline
\end{tabular}

Table 2: List of domain hits.

neurotoxins, cytotoxins and short toxins, and in other miscellaneous venom peptides (Table 2). The toxin acts by binding to the nicotinic acetylcholine receptors in the postsynaptic membrane of skeletal muscles and preventing the binding of acetylcholine, thereby blocking the excitation of muscles. This domain contains 60-75 amino acids that are fixed by 4-5 disulfide bridges and is nearly all beta sheet; it exists as either monomers or dimers.

\section{Conclusion}

Thirteen neurotoxin proteins of genus Naja from Elapidae family are summarized the identical regions. Using multiple sequences analysis and phylogenetic tree we observe the conserved residues to specify the evolutionary history and analysing sequence structure relationship of neurotoxin among Naja species. Efficient utilization of Polar, nonpolar, positively and negatively charged amino acids and their distribution in toxin sequence make them a killer element in snake venom. Comparative analyses specify that the neurotoxin demonstrates how proteins are generated within the nature's testing ground for tailormade biologic needs. Evolutionary studies of neurotoxin sequence of Naja genus found the common ancestor of all the Naja species. In future, different neurotoxin may be converted in laboratories through protein engineering to design synthetic peptide vaccine that have a much positive role.

\section{References}

1. Habermehl GG, Habermehl G (1981). Venomous animals and their toxins New York. Springer Verlag : 81-83.

2. Golay P, Smith HM, Broadley DG, Dixon JR, McCarthy C, et al. (1993) Endoglyphs and Other Major Venomous Snakes of the World A checklist. Azemiops herpetological data center : 478.

3. Kelly C, Barker N, Villet M, Broadley D (2009). Phylogeny, biogeography and classification of the snake superfamily Elapoidea: a rapid radiation in the late Eocene. Cladistics 25: 38-63.

4. Wallach V, Wuster W, Broadley DG (2009). In praise of subgenera: taxonomic status of cobras of the genus Naja Laurenti (Serpentes: Elapidae). Zootaxa 2236: 26-36.

5. Endo T, Tamiya N (1987). Current view on structure function relationship of postsynaptic neurotoxins from snake venoms. Pharmacol Ther 34: 403-451.

6. Yang CC (1974). Chemistry and evolution of toxins in snake venoms. Toxicon 12: $1-43$.

7. Rees B, Bilwes A (1993). Three-dimensional structures of neurotoxins and cardiotoxins. Chem Res Toxicol 6: 385-406.

8. TsetlinV I, Hucho F (2004). Snake and snail toxins acting on nicotinic acetylcholine receptors: fundamental aspects and medical applications. FEBS Lett 557: 9-13.

9. Rossetto O, Rigoni M, Montecucco C (2004). Different mechanism of blockade of neuroexocytosis by presynaptic neurotoxins. Toxicol Lett 149: 91-101.

10. Dufton MJ, Hider RC (1983). Conformational properties of the neurotoxins and cytotoxins isolated from Elapid snake venoms. CRC Crit Rev Biochem 14: 113171.

11. Durbin R, Eddy S, Krogh A and Mitchison G (2002). Biological Sequence Analysis: Probabilistic Models of Proteins and Nucleic Acids. Cambridge University Press, UK

12. Fry BG (2005). From genome to "venome": molecular origin and evolution of the snake venom proteome inferred from phylogenetic analysis of toxin sequences and related body proteins. Genome Res 15: 403-420.

13. Chang L, Lin S, Wang J, Hu WP, Wu B, et al. (2000) Structure-function studies on Taiwan cobra long neurotoxin homolog. Biochim Biophys Acta 1480: 293 301.

14. Gomase VS, Waghmare SB, Jadhav BV, Kale KV (2009). Functional analyses of the binding ability of neuro peptide $f(N P F)$ form Moniezia expansa. Gene Therapy and Molecular Biology 13: 64-70.

15. Gomase VS, Kale KV, Tagore S, Hatture SR (2008). Proteomics: Technologies for Protein Analysis. Current Drug Metabolism. 9: 213-220.

16. http://www.ncbi.nlm.nih.gov

17. (2013) Database resources of the National Center for Biotechnology Information. Nucleic Acids Res 41: D8-D20.

18. Papadopoulos JS, Agarwala R (2007). COBALT: constraint-based alignment tool for multiple protein sequences. Bioinformatics 23: 1073-1079.

19. Desper R, Gascuel O (2004). Fast and accurate phylogeny reconstruction algorithms based on the minimum-evolution principle. Mol Biol Evol 21: 587 598.

20. Saitou N, Nei M (1987). The neighbor-joining method: a new method for reconstructing phylogenetic trees. Mol Biol Evol 4: 406-425

21. Grishin NV (1995). Approximates the same model as Kimura, but can be computed for fraction of mismatched amino acids larger than 0.75 . J Mol Evol 41: $675-679$.

22. Grishin NV (1995). More general evolutionary model: substitution rates vary for both amino acids and sites. J Mol Evol 41: 675-679.

23. Gomase VS and Tagore S (2009). Phylogenomics: evolution and genomics intersection. Int J Bioinformatics Research and Applications 5: 548-563. 
Citation: Sherkhane AS, Gomase VS (2014) Evolutionary Distance and Conserved Domain Analysis of Divergent Phylogenetic Lineages from Genus Naja. J Data Mining Genomics Proteomics 5: 156. doi:10.4172/2153-0602.1000156

Page 6 of 6

24. Waghmare S, Waghmare D, Bhatnagar PS (2013). Species Diversity of Short Horned Grasshopper (Orthoptera: Acrididae) in Selected Grasslands of Solapur District, Maharashtra, India. J Biodivers Endanger Species 1: 110.

25. Marchler-Bauer A, Lu S, Anderson JB, Chitsaz F, Derbyshire MK, et al. (2011) "CDD: a Conserved Domain Database for the functional annotation of proteins." "Nucleic Acids Res 39: 225-229.
26. Marchler-Bauer A, Anderson JB, Chitsaz F, Derbyshire MK, DeWeese Scott C, Fong JH, et al. (2009) „CDD: specific functional annotation with the Conserved Domain Database." Nucleic Acids Res 37: 205-210. 